\title{
Contrast analysis between the trajectory of the planetary system and the periodicity of solar activity
}

\author{
Wei Sun ${ }^{1,2,3}$, Jian Wang ${ }^{1,2}$, JinRu Chen ${ }^{1,3}$, Ying Wang ${ }^{4}$, GuangMing $\mathbf{Y u}^{2}$, and XianHai $\mathrm{Xu}^{2}$ \\ ${ }^{1}$ College of Mathematics/College of Geography, Nanjing Normal University, Nanjing, 210023, China \\ ${ }^{2}$ School of Resources and Environment, Anqing Normal University, Anqing, 246133, China \\ ${ }^{3}$ Jiangsu Center for Collaborative Innovation in Geographical Information Resource Development and Application, \\ Nanjing, 210023, China \\ ${ }^{4}$ School of Astronomy and Space Science and Key Laboratory of Modern Astronomy and Astrophysics in Ministry of \\ Education, Nanjing University, Nanjing, 210039, China \\ Correspondence to: Jian Wang (jwang169@vip.sina.com)
}

Received: 11 September 2016 - Revised: 23 November 2016 - Accepted: 12 April 2017 - Published: 17 May 2017

\begin{abstract}
The relationship between the periodic movement of the planetary system and its influence on solar activity is currently a serious topic in research. The kinematic index of the planet juncture index has been developed to find the track and variation of the Sun around the centroid of the solar system and the periodicity of solar activity. In the present study, the kinematic index of the planetary system's heliocentric longitude, developed based on the orbital elements of planets in the solar system, and it is used to investigate the periodic movement of the planetary system. The kinematic index of the planetary system's heliocentric longitude and that of the planet juncture index are simulated and analyzed. The numerical simulation of the two kinematic indexes shows orderly orbits and disorderly orbits of 49.9 and 129.6 years, respectively. Two orderly orbits or two disorderly orbits show a period change rule of 179.5 years. The contrast analysis between the periodic movement of the planetary system and the periodicity of solar activity shows that the two phenomena exhibit a period change rule of 179.5 years. Moreover, orderly orbits correspond to high periods of solar activity and disorderly orbits correspond to low periods of solar activity. Therefore, the relative movement of the planetary system affects solar activity to some extent. The relationship provides a basis for discussing the movement of the planetary system and solar activity.
\end{abstract}

Keywords. History of geophysics (solar-planetary relationships)

\section{Introduction}

The movement of planets, solar activity, and global climate change are increasingly being explored. The periodicity of solar activity along with the physical mechanism of the changing of the Sun is an important topic in solar physics. Meanwhile, global climate change caused by planet movement and solar activity is an important subject in geophysics. McCracken (2014) and Wilson et al. (2008) found that solar activity is related to the movement of the planetary system. Charvátová (1990), Charvátová and Střeštík (2007), Scafetta et al. (2016), and Yndestad and Solheim (2017) studied the relation between the movement of the Sun and solar activity. Jager and Versteegh (2005) also discussed the link between the movement of the planetary system and solar activity. Since its introduction by Liu and Wang (2013), the planet juncture index has been used to study the period change rule of the Sun's rotation around the solar system's barycenter. Yang and Yang (2013) and Li et al. (2010) used this index and found an 11-year period for the sunspot and a 22-year period for the magnetic solar activity (Qu, 2007). Yang et al. (2010) and Zhao et al. (2014) conducted much research on the sunspot cycle. Friis-Christensen and Lassen (1991), Haigh (1996), Sharp (2013), Mörner (2010), Zhao and Feng (2014), Liu et al. (2011), and Zhao et al. (2011) successfully investigated the changes in surface environment and global climate on the basis of solar activity. Qu et al. (2004) put forth a similar theory of the changes in surface environment and global climate with solar activity. Several theories have 
Table 1. Orbital elements of planets in the solar system and the dwarf planet Pluto.(January 2000).

\begin{tabular}{|c|c|c|c|c|c|c|}
\hline Name & $\begin{array}{r}\text { Semimajor axis } \\
a(\mathrm{AU})\end{array}$ & $\begin{array}{r}\text { Eccentricity } \\
e\end{array}$ & $\begin{array}{r}\text { Orbital inclination } \\
i\left(\left(^{\circ}\right)\right.\end{array}$ & $\begin{array}{l}\text { Longitude of ascending node } \\
\Omega\left({ }^{\circ}\right)\end{array}$ & $\begin{array}{r}\text { Argument of pericenter } \\
\omega\left(^{\circ}\right)\end{array}$ & $\begin{array}{r}\text { Mean anomaly } \\
\lambda\left(\left(^{\circ}\right)\right.\end{array}$ \\
\hline Mercury & 0.38709893 & 0.20563069 & 7.00487 & 48.33167 & 77.45645 & 252.25084 \\
\hline Venus & 0.72333199 & 0.00677323 & 3.39471 & 76.68069 & 131.53298 & 181.97973 \\
\hline Earth & 1.00000011 & 0.01671022 & 0.00005 & -11.26064 & 102.94719 & 100.46435 \\
\hline Mars & 1.52366231 & 0.09341233 & 1.85061 & 49.57854 & 336.04084 & 355.45332 \\
\hline Jupiter & 5.20336301 & 0.04839266 & 1.30530 & 100.55615 & 14.75385 & 34.40438 \\
\hline Saturn & 9.53707032 & 0.05415060 & 2.48446 & 113.71504 & 92.43194 & 49.94432 \\
\hline Uranus & 19.19126393 & 0.04716771 & 0.76986 & 74.22988 & 170.96424 & 313.23218 \\
\hline Neptune & 30.06896348 & 0.00858587 & 1.76917 & 131.72169 & 44.97135 & 304.88003 \\
\hline Pluto & 39.48168677 & 0.24880766 & 17.14175 & 110.30347 & 224.06676 & 238.92881 \\
\hline
\end{tabular}

Note: These are basic data from NASA.

been developed for the mechanisms of cyclical variations in solar activity. The similar periodic trends of the movement of the Sun and solar activity are currently attracting considerable attention in academia (Jose, 1965; Damon et al., 1986, 1989; Tlatov, 2007). The movement of the planetary system's barycenter and the rotation of the Sun's barycenter about the solar system's barycenter show the same significant periods. The use of the periodic movement of the planetary system to study the periodic changes of the movement of the Sun or solar activity is also being increasingly investigated (Sun et al., 2017). Liu and Wang (2014) adopted the planet juncture index as the kinematic index because of its advantage in characterizing planetary conjunctions and separations. The planet juncture index plays a role in the periodic movement of the Sun and solar activity. Therefore, a certain relationship exists between the movement of the planetary system, solar activity, and global climate change. The movement of the planetary system can thus be used to interpret the periodic trends of the movement of the Sun and global climate change.

Previous scholars have studied solar activity by planetary conjunctions with an approximate method; they found that a tangible indicator must be provided for planetary conjunctions (Yang, 1991; Chen, 1996). An approximate method is efficient for a few planets but ineffective for many. Liu et al. (2013a, b) developed the kinematic index of the planet juncture index. Since then, the method has been used to characterize the degree of conjunctions and separations, physical characteristics and changing processes of planets. Although possibly advantageous in characterizing planetary conjunctions and separations, the planet juncture index cannot determine the specific azimuth of the planetary system's barycenter. The planetary system's heliocentric longitude can be used for an accurate solution to this problem. The present study aims to construct the kinematic index of the planetary system's heliocentric longitude for studying the exact position of the planetary system's barycenter and the period change rule of the planetary system's heliocentric longitude. A numerical simulation is conducted using certain analysis soft- ware, and the obtained essential features and change rules of the planetary system around the Sun are analyzed. Significant relationships are found between the movement of the planetary system and the essential features of solar activity. These findings provide a date basis and a strong theoretical basis for future research. The study provides a new way of and idea for studying solar activity.

\section{Constructing the kinematic index of the planetary system's heliocentric longitude}

\subsection{Determining the planetary system's barycenter}

Table 1 shows that each planet's orbit inclination is very small. The planets' orbit inclinations are all less than $3.5^{\circ}$, except for Mercury and the dwarf planet Pluto. Therefore, the datum is perpendicular to the plane of total rotational momentum for the solar system. Given that the planet orbit eccentricities are small, except for Pluto and Mercury, the planets' orbits are nearly circular in shape. Furthermore, the direction of the planet movement is in accordance with the spins of the Sun on its axis. To study the movement index of the planetary system, all the planet orbits are projected on the datum plane (Dai, 1979). The projection of the Sun on the datum plane is the coordinate origin and the projection of the planet orbits on the datum plane is nearly concentric circular in shape (Fig. 1). The planet juncture index is the total vector norm of the quality vector weights of the planetary system. The planetary system's heliocentric longitude is the included angle between the total quality-vector weights of the planetary system and the datum.

Figure 1 shows that the planets are the mass center, and the projections of planet orbits on the datum plane are nearly circular in shape. In the map, $P_{1}, P_{2}, P_{3}, \ldots P_{n}$ are the tail of the planet's quality vector weights; $m_{1}, m_{2}, m_{3}, \ldots m_{n}$ are the planet's mass; $\boldsymbol{r}_{1}, \boldsymbol{r}_{2}, \boldsymbol{r}_{3}, \ldots \boldsymbol{r}_{n}$ are the vector radii; $\varphi_{1}, \varphi_{2}, \varphi_{3}, \ldots \varphi_{N}$ are the individual planets' heliocentric longitudes; and $\varphi$ is the heliocentric longitude of the planetary system. Therefore, the vector $\left(\boldsymbol{r}_{\mathrm{s}}\right)$ between the planetary sys- 




Figure 1. Sketch map of planetary rendezvous.

tem's barycenter $(P)$ and the Sun's barycenter $(S)$ can be approximated as follows (Zhou, 1986):

$\boldsymbol{r}_{\mathrm{S}}(t)=\boldsymbol{s} \boldsymbol{p}(t)=\sum_{i=1}^{n} m_{i} \boldsymbol{r}_{i}(t) / \sum_{i=1}^{n} m_{i}$

where $\boldsymbol{r}_{\mathrm{s}}$ is the vector between the planetary system's barycenter and the solar system's barycenter. The beginning of the vector is the Sun's barycenter and the end of the vector is the planetary system's barycenter. To study the contribution of the planets to the planet's juncture index and heliocentric longitude, the average radius of the planet orbit is translated into quality-radius weights by Eq. (1), as illustrated in Table 2. The planet's vector represents a vector that starts at the Sun's barycenter and points towards the planet's barycenter. The planetary system's vector is determined by the planet's vector and mass. Each planet's radius weight $\left(\boldsymbol{r}_{i}\right)$ is defined as the product of its orbital radius and its mass fraction of the planetary system. Table 2 shows that Jupiter, Saturn, Uranus, and Neptune are the major contributors to the position of the planetary system's mass center because of their large quality-radius weights, whereas the rest of the planets contributed slightly because of their small quality-radius weights.

\subsection{Constructing the kinematic index of the planet juncture index}

The planetary system's barycenter vector is the total qualityvector weights of the planetary system, as obtained by Eq. (1). According to this principle, the planet juncture index is the norm of the total quality-vector weights for the planetary system. The spatial expression of the planet juncture index $(K)$ can then be obtained as follows:



Figure 2. Planet juncture index and change rate of the planetary system's mass center $(P)$ orbiting the fixed point of the Sun $(S)$.

$K(t)=\sqrt{\left[\sum_{i=1}^{n} \boldsymbol{r}_{i x}(t)\right]^{2}+\left[\sum_{i=1}^{n} \boldsymbol{r}_{i y}(t)\right]^{2}+\left[\sum_{i=1}^{n} \boldsymbol{r}_{i z}(t)\right]^{2}}$,

where $K$ is the planet juncture index; $\boldsymbol{r}_{i x}, \boldsymbol{r}_{i y}$, and $\boldsymbol{r}_{i z}$ are the projections of the total vector onto the three axes; and $t$ is the time. When all planets nearly unite, the planet juncture index can reach a maximum value; otherwise, the planet juncture index can reach a minimum value. Therefore, the planet juncture index represents the distance between the planetary system's barycenter and the Sun's barycenter. This index also represents the distance between the Sun's barycenter and that of the solar system. The index can also represent the degree of convergence and separation.

Table 1 shows the date of the orbital elements of planets in January 2000 obtained by NASA. Table 2 shows the astronomical data of planets. The calculations using $N$-body integration equations consider the orbital inclination and orbital eccentricity of planets and the gravitational interaction among the planets. In this study, the planet juncture index is obtained by numerical simulation using Eq. (2). Figure 2 shows the planet juncture index and its change rate.

The planet juncture index is the norm of the total qualityvector weights for the planetary system, and its basic unit is the astronomical unit. The acceptable values for the planet juncture index range from 0 to $7.5 \mathrm{AU}$ (Liu and Wang, 2014). When the planets lie on the same side of the Sun with the same heliocentric longitude, the planet juncture index can reach a maximum value and the degree of juncture can be high. Otherwise, the planet juncture index reaches a minimum value and the degree of juncture is low. The planetary system's barycenter, the Sun's barycenter, and the solar system's barycenter overlap at the moment. In theory, the maximum value of the planet juncture index is 7.520204 when the planets lie on the same side of the Sun. In reality, the 
Table 2. Astronomical data of planets.

\begin{tabular}{lrrrrrr}
\hline Name & $\begin{array}{r}\text { Period } \\
(\mathrm{d})\end{array}$ & $\begin{array}{r}\text { Mass } \\
(\mathrm{kg})\end{array}$ & $\begin{array}{r}\text { Average orbit radius } \\
(\mathrm{km})\end{array}$ & $\begin{array}{r}\text { Astronomical unit } \\
(1 \mathrm{AU})\end{array}$ & $\begin{array}{r}\text { Radius weights } \\
\left(\boldsymbol{r}_{i}\right)\end{array}$ & $\begin{array}{r}\text { Angular velocity } \\
(\omega)\end{array}$ \\
\hline Mercury & 87.969 & $0.3301 \times 10^{24}$ & 56672443.84 & 0.38709893 & $4.69091 \mathrm{E}-05$ & 26.08840762 \\
Venus & 224.701 & $4.8676 \times 10^{24}$ & 108207517.8 & 0.72333199 & 0.001320004 & 10.21344422 \\
Earth & 365.256 & $5.9726 \times 10^{24}$ & 149579112 & 1.00000011 & 0.002238882 & 6.2831853 \\
Mars & 686.98 & $0.64174 \times 10^{24}$ & 226923421.8 & 1.52366231 & 0.000363432 & 3.340666584 \\
Jupiter & 4332.587 & $1898.3 \times 10^{24}$ & 777657818.7 & 5.20336301 & 3.699505551 & 0.529699953 \\
Saturn & 10759.22 & $568.36 \times 10^{24}$ & 1431426696 & 9.53707032 & 2.038821640 & 0.213302742 \\
Uranus & 30685.4 & $86.816 \times 10^{24}$ & 2869262907 & 19.1912393 & 0.624274378 & 0.074790328 \\
Neptune & 60189.0 & $102.42 \times 10^{24}$ & 4494894315 & 30.48168677 & 1.153633570 & 0.038129411 \\
Pluto & 90465.0 & $0.0131 \times 10^{24}$ & 5720641564 & 39.48168677 & $1.88377 \mathrm{E}-04$ & 0.025368608 \\
\hline
\end{tabular}

Note: These are basic data from NASA.

value of the planet juncture index is usually less than the maximum value because the planet heliocentric longitudes are rarely uniform. Figure 2 shows the planet juncture index and change rate of the planetary system. Figure 2A1 shows that the planet juncture index lasts for 1000 years from 1000 to $2000 \mathrm{AD}$. The diagram shows that the periodic trend of the planet juncture index is relatively obvious. Among the significant trends, the cycle of the maximum or minimum value of planet juncture index is about 19.8585 years. Similar trend is observed in the periodic trend of the solar magnetic field corrected by Liu (2013a, b). A period change rule of a 179.5-year cycle is observed. This period change rule is consistent with the change that takes place once every 2 centuries (about 160 to 210 years) for solar activity and global climate change. Figure $2 \mathrm{~A} 2$ shows that the change rate of the planet juncture index lasts about 1000 years from 1000 to $2000 \mathrm{AD}$. The diagram shows an obvious 179.5-year periodic change rate of the planet juncture index. This period change rule is also consistent with the double-century period. The period showing small variation in the planet juncture index is about 49.9 years. This period corresponds to the highly uniform sections of the planet juncture index (enclosed in red boxes). The large variation in the planet juncture index is about 129.6 years. This period corresponds to the nonuniform sections of the index. The uniform sections correspond to the planets' orderly orbits and the nonuniform sections correspond to the planet's disorderly orbits. The periodic movement of the planetary system's barycenter into the orderly orbit or the disorderly orbit is about 179.5 years. This remarkable period is scientifically important for studying the movement of the planetary system. The positions of the prolonged minima of solar activity are shown in Fig. 2. In the figure, O, M, W, S, $\mathrm{Ma}$, and D represent the Oort minimum (1040-1080 AD), Medieval Minor minimum (1150-1200 AD), Wolf minimum (1270-1350 AD), Spörer minimum (1430-1520 AD), Maunder minimum (1620-1710 AD), and Dalton minimum (1787-1843 AD), respectively. Figure 2a1, a2, a3, a4, a5, and a6 show the planetary system's orderly orbits. Figure 2b1, b2, $\mathrm{b} 3, \mathrm{~b} 4, \mathrm{~b} 5$, and b6 present the planetary system's disorderly orbits. As shown in Fig. 2, the planet juncture index exhibits marked periodic variations. The planet juncture index may be advantageous in representing the distance between the planetary system's barycenter and the Sun's barycenter, as well as the degree of planet conjunctions and separations. However, the index cannot be used in representing the change of the planetary system's barycenter orbit without considering the planetary system's heliocentric longitude. The planetary system's heliocentric longitude is an important indicator of the movement of the planetary system. Therefore, the planetary system's heliocentric longitude must be constructed.

\subsection{Constructing the kinematic index of the planetary system's heliocentric longitude}

The planetary system's heliocentric longitude is the angle between the line from the projection of the planetary system's barycenter onto the datum to the Sun's barycenter and the datum axis (Fig. 1). The planetary system's heliocentric longitude is denoted as $\varphi$ and expressed as follows:

$\sin \varphi(t)=\left|\sum_{i=1}^{n} \boldsymbol{r}_{i y}(t)\right| /\left|\sum_{i=1}^{n} \boldsymbol{r}_{i x y}(t)\right|$.

In this study, the planetary system's heliocentric longitude $(\varphi)$ is given by

$\varphi(t)=k \pi \pm \arcsin \left(\left|\sum_{i=1}^{n} \boldsymbol{r}_{i y}(t)\right| /\left|\sum_{i=1}^{n} \boldsymbol{r}_{i x y}(t)\right|\right)(k=0,1,2)$,

where $\varphi$ is the planetary system's heliocentric longitude, $k$ is constant, $\boldsymbol{r}_{i y}$ is the component vector on the $y$ axis, $\boldsymbol{r}_{i x y}$ is the sum vector on the $x$ axis and $y$ axis, and $t$ is the year. The dates in Table 1 or 2 and the gravitational interaction among the planets are considered in conducting the $N$-body integration. Accordingly, the different years of the planetary 


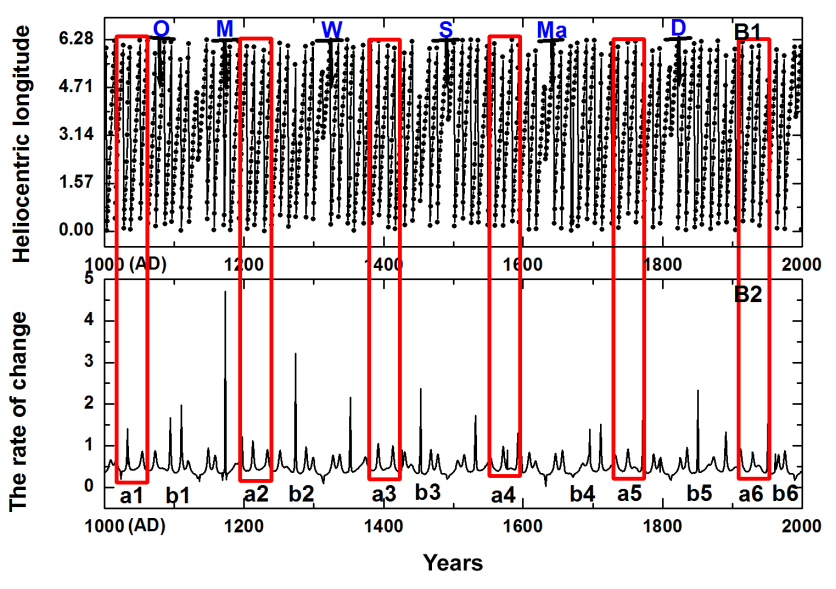

Figure 3. Planetary system heliocentric longitude and change rate of the planetary system's mass center $(P)$ orbiting the fixed point of the Sun $(S)$.

system's heliocentric longitude can be obtained for studying the periodic trends by numerical simulation using Eq. (4). Figure 3 shows the planetary system's heliocentric longitude and its change rate.

The planetary system's heliocentric longitude is the exact location of the planetary system's barycenter, which is located next to the Sun's barycenter. The planetary system's heliocentric longitude unit is in radians. The acceptable values for the planetary system's heliocentric longitude range from 0 to $2 \pi$. Figure 3 shows the planetary system's heliocentric longitude and its change rate over time. The graph's horizontal axis shows the time range (1000-2000 AD), and the vertical axis shows the planetary system's heliocentric longitude and its change rate. Figure 3B1 shows the planetary system's heliocentric longitude over time from 1000 to $2000 \mathrm{AD}$, whilst Fig. 3B2 shows the corresponding change rates. The planetary system's heliocentric longitude shows a periodical change range of $0-2 \pi$ (Fig. 3B1). The planetary system's heliocentric longitude has a period of 11.5 years, indicating that the planetary system's barycenter takes 11.5 years to move around the Sun. The sunspot has a roughly 11-year cycle. The planetary system's heliocentric longitude shows an obvious periodic change rate of 179.5 years (Fig. 3B2). This period corresponds with the cycle of the planetary system's heliocentric longitude in Fig. 3B1. Figure 3B2 shows that the planetary system's heliocentric longitude has a periodic change rate of about 19.8 years from 1000 to $2000 \mathrm{AD}$. The periodic changes of the planetary system's heliocentric longitude in Fig. 3 agree well with those in Fig. 2. Meanwhile, the maximum or minimum change rate has a period of roughly 179.5 years. This period is similar to the double-century period in solar activity and global climate change. These cycles can be found in the planet's juncture index and heliocentric longitude. Therefore, the planetary system's heliocentric longitude complements the deficiency of a short cycle and pro-

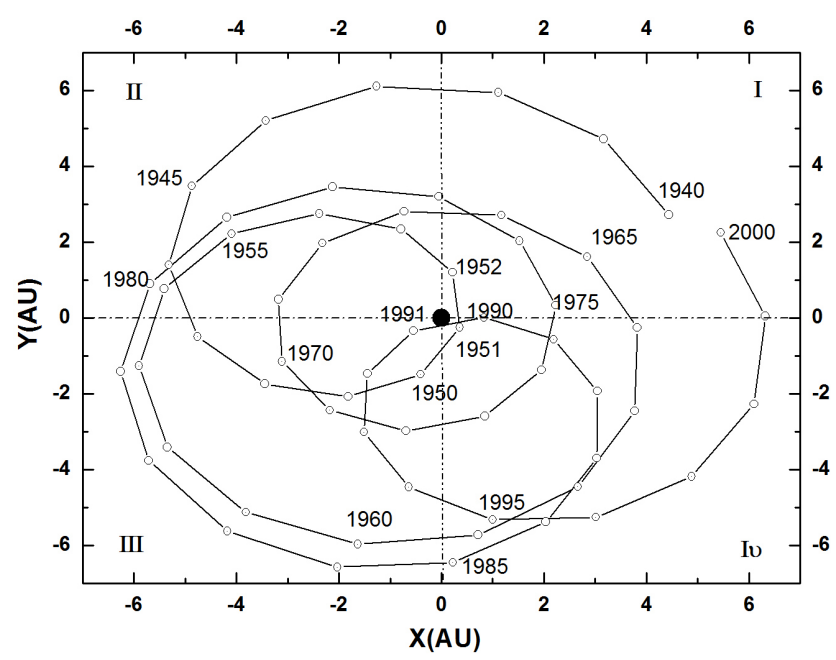

Figure 4. Heliocentric longitude of the planetary system's mass center.

vides reference for studying the planet juncture index. The graph of the planetary system's heliocentric longitude directly reflects the location and variation characteristics of the planetary system's barycenter. The periodic changes of the planetary system's heliocentric longitude can symbolize the periodic changes of the planetary system's barycenter around the Sun or the Sun around the solar system's barycenter, to some extent. The change rate of the planetary system's heliocentric longitude reflects the movement of the planetary system's barycenter at the rate of change level. The value of the planetary system's heliocentric longitude can increase when the planetary system's barycenter is near the Sun's barycenter. The planetary system's heliocentric longitude reaches a maximum value when the planetary system's mass center coincides with that of the Sun. The relationships among the planetary system's barycenter, the Sun's mass center, and the solar system's barycenter and its period trends are studied according to the planetary system's heliocentric longitude. The kinematic indexes of the planetary system's heliocentric longitude and the planet juncture index are simulated by $N$ body integration. This method is useful for further studying the variation characteristics of the movement of the planetary system's barycenter around the Sun's barycenter or the Sun's barycenter around the solar system's barycenter. Figure 3 shows that the planetary system's heliocentric longitude and its change rate vary all the time. The details on the planetary system's heliocentric longitude are presented in Fig. 4.

Figure 4 shows the change rate of the planetary system's heliocentric longitude over time from 1940 to 2000 AD. Accordingly, the position and movement of the planetary system's barycenter from 1940 to $2000 \mathrm{AD}$ is precisely measured. The acceptable values for the planetary system's heliocentric longitude range from 0 to $2 \pi$. These values are divided into four quadrants: the first quadrant is from 0 to $0.5 \pi$, the second quadrant is from $0.5 \pi$ to $1 \pi$, the third 
quadrant is from $1 \pi$ to $1.5 \pi$ and the fourth quadrant is from 1.5 to $2 \pi$ (Fig. 4). In Fig. 4, the hollow circle represents the position of the planetary system's barycenter and the solid black circle represents the solar interior at the origin coordinates $(0,0)$. The planet juncture index represents the distance between the planetary system's barycenter and the solar interior, and the planetary system's heliocentric longitude is the angle between the line from the projection of the planetary system's barycenter onto the datum to the Sun's barycenter and the datum axis. The planetary system's barycenter changes yearly from 1940 to 2000 AD. The planet juncture index and the planetary system's heliocentric longitude also change accordingly. This conclusion corresponds to the change rules shown in Figs. 2A1 and 3B1. The planetary system's barycenter is far from the solar interior, with a high value of the planet juncture index and a low value of the variation in its heliocentric longitude. The variation in the planetary system's heliocentric longitude is less than 0.523 each year during the periods of $1940-1945,1980$ 1985, and 1995-2000 AD. At this point, the planet juncture index is nearly the maximum. The planetary system barycenter is near the solar interior or it overlaps the low value of the planet juncture index and the high value of the variation in its heliocentric longitude. The variation in the planetary system's heliocentric longitude is nearly 1.57 each year during the period of 1950-1951 AD, over 1.57 during the period of 1951-1952 AD, and very close to 3.14 during the period of 1990-1991 AD. At this point, the planet juncture index is significantly small or nearly zero. Therefore, the planetary system's barycenter is far from the solar interior. Moreover, the variation in the planetary system's heliocentric longitude is small when the planet juncture index is high, and vice versa. Thus, the planet's juncture index and heliocentric longitude show similar periodic rules, and they can be used in studying the movement of the planetary system, solar activity, and global climate change.

\section{Trajectory of the planetary system movement and the periodic rule of solar activity}

The planet's juncture index and heliocentric longitude are two important kinematic indexes of the movement of the planetary system's barycenter around the Sun or the Sun around the solar system's barycenter. The effect of the planetary system movement on solar activity and global climate change is currently a serious concern in research on global climate change. The periodic rule of the planetary system movement and the use of the periodic trends of solar activity and global climate change are rarely investigated. The present numerical simulation has two advantages: one is the full consideration of the gravitational interaction among planets, and the other is the simulation of the planet's juncture index and heliocentric longitude by $N$-body integral equations. Nearly 1000 years of basic data about the planet juncture index and heliocentric longitude are obtained by numerical simulation. These data are used for studying the periodic rule of the planetary system's barycenter around the solar interior. The trajectory of the planetary system's barycenter shows 179.5 years of a double-century cycle consisting of 49.9 years of orderly orbit and 129.6 years of disorderly orbit. A similar cycle is found for solar activity.

\subsection{Periodic trends of the trajectory of the planetary system}

The planetary movement contributes to the unique regular periodic movement of the planetary system's barycenter around the Sun. The trajectory of the planetary system's barycenter presents different moving shapes. Figure 4 shows the periodic change rule of the planetary system's movement over time from 1007.8 to 2016.6 AD. The planet juncture index is the distance between any point of the trajectory and the center of the circle. Meanwhile, the planetary system's heliocentric longitude is the angle between the line from any point of the trajectory to the center of the circle and the datum axis. Two main kinds of the planetary system movement for nearly 1000 years are observed, namely, orderly orbit and disorderly orbit (Fig. 5). The trajectory of the planetary system's movement shows a trefoil-shaped orbit. Compared with other times, the change rates of the planet juncture index in orderly orbit and those of the planetary system's heliocentric longitude are smaller and remain stable at equal times. Conversely, the trajectory of the planetary system's movement looks chaotic. Both the planet juncture index and the planetary system's heliocentric longitude have an irregular rate of change over time, which we call a disorderly orbit. Figure 5a1, a2, a3, a4, a5, and a6 show orderly orbits, whilst Fig. 5b1, b2, b3, b4, b5, and b6 show disorderly orbits. Orderly orbit is also called trefoil-shaped orbit. The trajectory of the planetary system's movement shows alternating orderly orbits and disorderly orbits. The periods of orderly orbits are 50.5, 50.1, 49.8, 49.7, 49.8, and 49.7 years, as shown in Fig. 5a1, a2, a3, a4, a5, and a6, respectively. The periods of disorderly orbits are 129.4, 129.6, 129.7, 129.7, and 129.8 years, as shown in Fig. 5b1, b2, b3, b4, b5, and b6, respectively. The average periods for orderly and disorderly orbits are nearly 49.9 and 129.6 years, respectively. The trajectory of the planetary system's barycenter shows that the double-century cycle of 179.5 years can consist of 49.9 years of orderly orbit and 129.6 years of disorderly orbit. The trajectory of the planetary system in Fig. 5 corresponds to the planet juncture index in Fig. 2 and the planetary system's heliocentric longitude in Fig. 3. As shown in Fig. 5a1, a2, a3, a4, a5, and a6, all the orderly orbits show a trefoil shape and are relatively stable. The distance from the planetary system's barycenter to the solar interior is relatively small and its change rate is also low. The planet juncture index is relatively small and its change rate is also low during orderly orbit periods. Meanwhile, the planetary system's heliocen- 


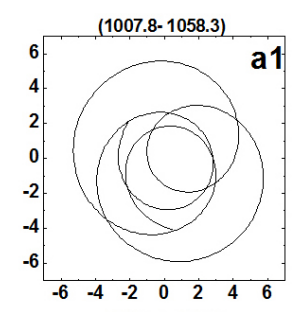

(1367.4-1417.2)
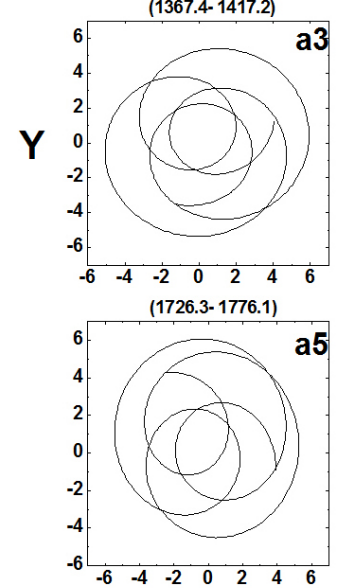

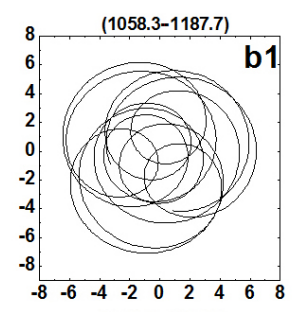

(1417.2-1546.9)



(1776.1-1905.9)

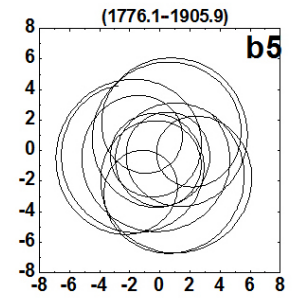

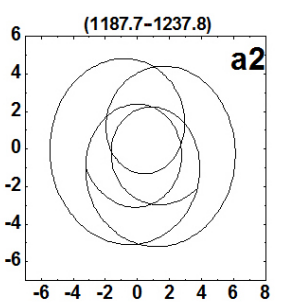

(1546.9-1596.6)

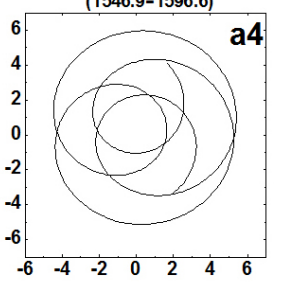

(1905.9-1955.6)



a6

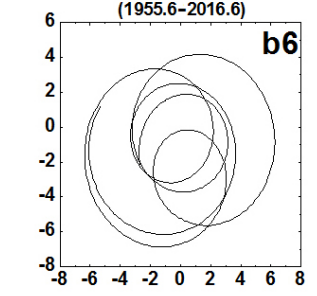

Figure 5. Life cycle diagrams of the trajectory of the planetary system from 1007.8 to 2016.6 AD.

tric longitude is relatively stable and its change rate is low. Figure 2 shows that the planet juncture index is relatively large and its change rate is low during orderly orbit periods. This trend corresponds to the trajectory of the planetary system in Fig. 5. Figure 3 shows that the planetary system's heliocentric longitude is relatively stable and its change rate is low during orderly orbit periods. This trend also corresponds to the trajectory of the planetary system in Fig. 5. Figure 5 shows that the large planet juncture index results in a low change rate of the planetary system's heliocentric longitude, as obtained by contrasting the planet's juncture index and heliocentric longitude, and vice versa. The movement of the planetary system exhibits certain regularity and periodicity. The planet juncture index has a distinct period of about 19.8 years, and the planetary system's heliocentric longitude has a distinct period of about 11.5 years. All of the planet juncture index, planetary system's heliocentric longitude, and trajectory of the planetary system have a doublecentury cycle of 179.5 years that consists of 49.9 years of orderly orbit and 129.6 years of disorderly orbit. These results are important for the research on solar activity.

\subsection{Contrast and analysis between the trajectory of the planetary system and the periodic rule of solar activity}

The relative number of sunspots was introduced by Wolf in 1848. Through collection, sorting, and analysis of data, more than 300 years of sunspot data are obtained and can be used to study solar activity. This section compares the number of sunspots and the trajectory of the planetary system to discover if they have the same regular patterns.

Figure 6 shows the trajectory of the planetary system in relation to the number of sunspots. According to the sunspot data since $1700 \mathrm{AD}$ and the trajectory of the planetary system since $1630 \mathrm{AD}$, the relationship between the sunspots and the trajectory of the planetary system is analyzed. Figure 6D1 shows the trajectory of the planetary system from 1630 to 2016 AD. The movement of the planetary system is divided into five time intervals of 1630.0-1726.3, 1726.3-1776.1, 1776.1-1905.9, 1905.9-1955.6, and 1955.6-2016.6 AD, as shown in Fig. 6d1, d2, d3, d4, and d5, respectively. Orderly and disorderly orbits are found in the five time intervals. The orderly orbits correspond to the time intervals shown in Fig. 6d1, d3, and d5, whilst the disorderly orbits correspond to the time intervals shown in Fig. $6 \mathrm{~d} 2$ and $\mathrm{d} 4$. Orderly orbit is also called trefoil-shaped orbit because of its cloverleaf shape. Figure $6 \mathrm{~d} 2$ and $\mathrm{d} 4$ show that the distance between the planetary system's barycenter and the Sun's barycenter displays periodic variation, and the orderly orbit lasts about 50 years. The planet's juncture index and heliocentric longitude are relatively smooth. Two orderly orbits are separated by 130 years. Figure $6 \mathrm{~d} 1$, d2, and d 3 show that the distance between the planetary system's barycenter and the Sun's barycenter exhibits no cyclic change. The planet's juncture index and heliocentric longitude look messy 



Figure 6. Contrast of the trajectory of the planetary system and solar activity.

for disorderly orbit. They reach their maximum or minimum value very easily. Two disorderly orbits are separated by 50 years. An orderly orbit takes about 180 years before realizing the next orderly orbit, and a similar trend is observed for disorderly orbit. This period is very similar to the doublecentury period. Figure 6D2 shows the number of sunspots from 1700 to $2016 \mathrm{AD}$. Two active phases are observed in the sunspot data of 316 years, that is, the periods of 1726.31776.5 and 1905.9-1955.6 AD (Fig. 6d6 and d7). The number of sunspots increases and is higher in two active phases (Fig. $6 \mathrm{~d} 6$ and d7) than the other periods. The solar active phase shows a high number of sunspots for 50 years and an interval of 130 years. About 180 years is needed before realizing the next active phase. The solar active phases in Fig. $6 \mathrm{~d} 6$ and $\mathrm{d} 7$ correspond to the orderly orbits in Fig. $6 \mathrm{~d} 2$ and $\mathrm{d} 4$. The low sunspot activity corresponds to the disorderly orbits in Fig. 6d1, d3, and d5. Figure 6 shows that the orderly orbit corresponds to strong solar activity and the disorderly orbit corresponds to weak solar activity. Thus, the movement of the planetary system affects solar activity to some extent.

Figure 7 shows the characteristics of the number of sunspots. The number of sunspots is the major target that describes the solar activity level. Sunspots are relatively cool and dark features on the surface of the Sun and are often roughly circular in shape. They appear as dark patches on the surface of the Sun and are caused by small areas of strong magnetic activity. Sunspots disrupt the normal flow of intensely heated gases. In the nearly 2 centuries since the 11 year sunspot cycle was discovered, scientists have struggled to predict the size of future maxima. The first sunspot cycle began in $1755 \mathrm{AD}$ and is currently in its 24th cycle. In the present study, the characteristic diagram of the sunspot cycles is obtained based on the number of sunspots since $1700 \mathrm{AD}$ (Fig. 7). The two periods of time enclosed in red boxes in Fig. $7 \mathrm{e} 1$ and $\mathrm{e} 2$ are the orderly orbits in the periods of 1726.3-1776.1 and 1905.9-1955.6 AD for the planetary system. Figure 7E1 shows the total number of sunspots. The total number of sunspots is relatively high and increases in the planetary system's orderly orbit, indicating that solar activity is present. The total number of sunspots is relatively small and sometimes reaches its minimum value in the planetary system's disorderly orbit, indicating that solar activity is not present. Figure 7E2 shows the average number of sunspots. The average number of sunspots is relatively high and increases during the planetary system's orderly orbit, indicating that solar activity is present. The average number of sunspots is relatively small and sometimes reaches its minimum value during the planetary system's disorderly orbit, indicating that solar activity is not present. The characteristics in Fig. 7E2 fit with those in Fig. 7E1. Figure 7E3 shows the standard deviation of the number of sunspots. The standard deviation of the number of sunspots is relatively high and increases in the planetary system's orderly orbit, indicating that solar activity is present. The standard deviation of the number of sunspots is relatively small and sometimes reaches its minimum value in the planetary system's disorderly orbit, indicating that solar activity is not present. The characteristics in Fig. 7E3 fit with those in Fig. 7E1 and E2. Figure 7E4 shows the number of years each sunspot period lasts. The 


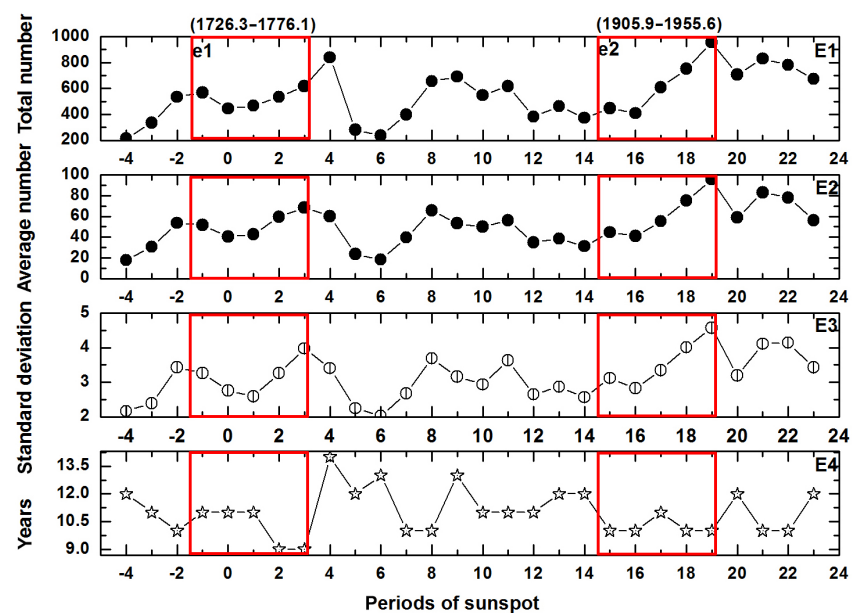

Figure 7. Characteristic diagram of the sunspot cycles.

number of years each sunspot period lasts remains relatively stable, with the average being about 10 years. These results indicate that the sunspot period has a relatively short lifetime during orderly orbit. The number of years each sunspot period lasts during disorderly orbit is relatively unstable and its maximum value can be up to 14 years. The average number of years each sunspot period lasts is 11.5 years. These findings indicate that the sunspot period is unstable during disorderly orbit. The characteristics in Fig. 7E4 fit with those in Fig. 7E1, E2, and E3. The characteristic diagrams of the sunspot cycles show that, during orderly orbit, the number of sunspots is relatively high, increases, and shows a high change rate. The sunspots also become active with short cycle times with an average of 10 years. Meanwhile, during disorderly orbit, the number of sunspots is relatively low, becomes nonexistent, and exits the solar minimum. The number of sunspots is also unstable, with long cycle times with an average range of 11.5-14 years. Figure 7 also shows that the active solar cycle takes 179.5 years before realizing the next active cycle.

Figure 8 shows the protracted solar minimum corresponding to the disorderly orbit of the planetary system. The Sun is headed into a grand minimum, that is, a period of unusually low solar activity. A relatively low number of or nonexistent sunspots are observed during the protracted solar minimum. Since $1000 \mathrm{AD}$, there have been six protracted solar minimums, namely, the Oort minimum (1040-1080 AD), Medieval Minor minimum (1150-1200 AD), Wolf minimum (1270-1350 AD), Spörer minimum (1430-1520 AD), Maunder minimum (1620-1710 AD), and Dalton minimum (1787-1843 AD). The six protracted solar minimums are shown in Fig. 8F1, F2, F3, F4, F5, and F6. As shown Fig. 7, all the orbits of the planetary system are in disorder during the six protracted solar minimums. The planet juncture index and heliocentric longitude are also in disorder during the six protracted solar minimums (Figs. 2 and 3). The legends O,
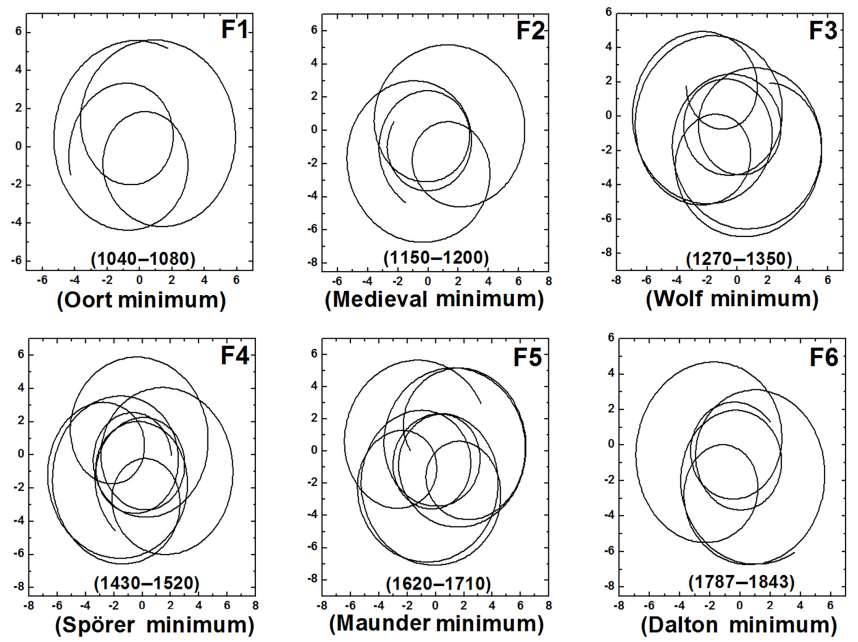

Figure 8. Prolonged minima of solar activity corresponding to the disorderly orbit of the planetary system.

M, W, S, Ma, and D in Figs. 2 and 3 are used to denote the positions of the six protracted solar minimums. These minimums correspond to the planetary system's disorderly orbits in Fig. 8. The planet juncture index, heliocentric longitude, and trajectory of the planetary system are analyzed and compared with solar activity. The numerical simulation results show a high number of sunspots and strong solar activity during the orderly orbit and a low number of sunspots, low solar activity, and the occurrence of a protracted solar minimum or little ice age during the disorderly orbit. Moreover, the periodic trends of the planet juncture index and heliocentric longitude are consistent, but certain differences between the two are also obtained. The movement of the planetary system remains consistent with the solar activity. The planetary system can reflect the strength of solar activity to some extent. Therefore, the planet's juncture index and heliocentric longitude are important parameters for studying the movement of the planetary system, thereby developing a new direction for studying solar activity with scientific and realistic value.

\section{Conclusions}

In this study, the kinematic index of the heliocentric longitude is developed based on the orbital elements of planets and is used to investigate the exact position of the planetary system over time. The numerical simulation results show a 19.8-year cycle for the planet juncture index and an 11.5-year cycle for the planetary system's heliocentric longitude. The use of the periodic rule of the planetary system's movement as a cross-subject research method is a new way of investigating solar activity.

The present study provides two innovations: one is the full consideration of the gravitational interaction among planets, and the other is the simulation of the planet's juncture in- 
dex and heliocentric longitude by $N$-body integral equations. Nearly 1000 data of the planetary system movement are obtained by numerical simulation. The movement of the planetary system from 1007.8 to $2016.6 \mathrm{AD}$ shows a 49.9-year cycle for orderly orbit and a 129.6-year cycle for disorderly orbit. A double-century cycle of about 179.5 years is necessary for an orderly orbit or disorderly orbit before realizing the next cycle. This trend is consistent with solar activity and global climate change. This finding is theoretically valuable for studying solar activity and global climate change. Charvátová (2000) has noted that the same phenomenon can be observed in the orbit of the Sun around the center of mass of the solar system. Our results reinforce previous findings by Charvátová.

The comparison between the planet movement and number of sunspots shows that, during orderly orbit, the number of sunspots is relatively high, increases, and shows a high change rate. Moreover, the sunspots become active with short cycle times with an average of 10 years. Meanwhile, during disorderly orbit, the number of sunspots is relatively small, becomes nonexistent, and exits the solar minimum. The number is also unstable with long cycle times with an average range of 11.5-14 years. These findings illustrate that the planet movement influences solar activity to some extent. Similar results have been obtained when Charvátová studied the orbit of the Sun around the solar system barycenter (Charvátová., 2000).

Six protracted solar minimums have been observed since $1000 \mathrm{AD}$, and these minimums are the Oort minimum (10401080 AD), Medieval Minor minimum (1150-1200 AD), Wolf minimum (1270-1350 AD), Spörer minimum (14301520 AD), Maunder minimum (1620-1710 AD), and Dalton minimum (1787-1843). The movement of the planetary system is simulated, and the results indicate that the solar minimum and little ice ages correspond to the planetary system's disorderly orbit. Therefore, the planetary system's disorderly orbit usually occurs with the protracted solar minimum.

The planet's juncture index and heliocentric longitude are two important kinematic indexes of the movement of the planetary system's barycenter around the solar system's barycenter. The planet juncture index represents the distance between the planetary system's barycenter and the Sun's barycenter. This index also represents the degree of convergence and separation of the planetary system. The planetary system's heliocentric longitude represents the exact location of the planetary system's barycenter relative to the solar system's barycenter. The planetary system's heliocentric longitude also complements the deficiency of a short cycle and provides reference for studying the planet juncture index. The movement of the planetary system remains consistent with solar activity to some extent. Thus, analyzing the periodic trends in the planet movement can help in comprehensively understanding the periodic trends in solar activity and global climate change.
Data availability. Data used for the orbital elements of planets in the solar system are available from NASA (https://nssdc.gsfc.nasa. gov/planetary/).

Competing interests. The authors declare that they have no conflict of interest.

Acknowledgements. This work was supported by the Project Funded by the Priority Academic Program Development of Jiangsu Higher Education Institutions (164320H116), and the Project Funded by the Education Department Natural Science of Anhui Province (KJ2017A371, AQKJ2015B021). The authors thank the reviewers for their suggestions and ideas during this article's review process.

Edited by: M. Temmer

Reviewed by: G. Verbanac and one anonymous referee

\section{References}

Charvátová, I.: On the relation between solar motion and solar activity in the years 1730-80 and 1910-60, Bull. Astron. Inst. Czechosl., 41, 200-204, 1990.

Charvátová, I.: Can origin of the 2400-year cycle of solar activity be caused by solar inertial motion?, Ann. Geophys., 18, 399-405, doi:10.1007/s00585-000-0399-x, 2000.

Charvátová, I. and Střeštík, J.: Relations between the solar inertial motion, solar activity and geomagnetic index aa since the year 1844, Adv. Space Res., 40, 1026-1031, 2007.

Chen, X. Z.: Solar activity modulation by planetary perturbations, Chin. Astron. Astrophys., 16, 207-212, 1996.

Dai, W. S.: The Development of Solar System (Rudin), Shanghai, Shanghai Scientific \& Technical Publishers, 12, 22-23, 1979.

Damon, P. E., Cheng, S., and Linick, T. W.: Fine and hyperfine structure in the spectrum of secular variations of atmospheric ${ }^{14}$ C, Radiocarbon, 31, 704-718, 1989.

Damon, P. E. and Linick, T. W.: Geomagnetic-heliomagnetic modulation of atmospheric radiocarbon production, Radiocarbon, 28, 266-278, 1986.

Friis-Christensen, E. and Lassen, K.: Length of the solar cycle: An indicator of solar activity closely associated with climate, Science, 254, 698-700, 1991.

Haigh, J. D.: The impact of solar variability on climate, Science, 61, 981-984, 1996.

Jager, C. D. and Versteegh, G. J. M.: Do planetary motions drive solar variability?, Sol. Phys., 229, 175-179, 2005.

Jose, P. D.: Sun's Motion and Sunspots, Astron J., 70, 193-200, 1965.

Li, K. J., Feng, W., and Liang, H. F.: The abnormal 24th solar cycleThe first complete solar cycle of the new millennium, Sci. Sinica, 40, 1293-1301, 2010.

Liu, F. G. and Wang, J.: Changes of the planet juncture index and solar revolution cycle around the mass center of the solar system, Chinese J. Geophys., 56, 1457-1466, 2013. 
Liu, F. G. and Wang, J.: The 22-year cycle of solar revolution round the mass center and its relation with the solar activity. Chinese J. Geophys., 57, 3834-3840, 2014.

Liu, F. G., Wang, J., Bai, S. B., and Shang, Z. Y: The planetary motion and the change of 22-year cycle of the Sun spin velocity, Prog. Geophys., 28, 1678-1683, 2013a (in Chinese).

Liu, F. G., Wang, J., Shang, Z. Y., and Zheng, Y.: Study on longterm cyclical rhythm of solar activity, Chin. J. Prog. Geophys., 2, 570-578, 2013b.

Liu, L. B., Wan, W. X., Chen, Y. D., and Le, H. J.: Solar activity effects of the ionosphere: A brief review, Chin. Sci. Bull., 56, 1202-1211, 2011.

McCracken, K. G., Beer, J., and Steinhilber, F.: Evidence for planetary forcing of the cosmic ray intensity and solar activity throughout the past 9400 years, Sol. Phys., 286, 609-627, 2014.

Mörner, N.-A.: Solar minima, Earth's rotation and little ice ages in the past and in the future, the north Atlantic-European case, Global Planet Change., 72, 282-293, 2010.

Qu, W. Z., Deng, S. G., Huang, F., Chen, L., and Zhao, X.: The influence of magnetic index abnormal change in solar magnetic field on climate at middle latitude of North Hemisphere, Chinese J. Geophys., 47, 448-456, 2004.

Qu, W. Z., Huang, F., Zhao, J. P., Deng, S. G., Liu, Y. C., and Li, S. J.: The significance of solar magnetic field direction variation on anomalous variability of the atmosphere temperature on the earth, Chinese J. Geophys., 50, 1304-1310, 2007 (in Chinese).

Scafetta, N., Milani, F., Bianchini, A., and Ortolani, S.: On the astronomical origin of the Hallstatt oscillation found in radiocarbon and climate records throughout the Holocene, Earth-Sci. Rev., 162, 24-43, 2016.

Sharp, G. J.: Are Uranus \& Neptune responsible for solar grand minima and solar cycle modulation?, Int. J. Astron. Astrophys., 3, 260-273, 2013.
Sun, W., Wang, J., Chen, J. R., Wang, Y., Liu, F. G., and Yu, G. M: Variations of the planet juncture index and heliocentric longitude with spectral analysis for approximately 2000 years, Chin. Sci. Bull., 62, 407-419, 2017.

Tlatov, A. G.: 22-year variations of the solar rotation and solar activity cycles, Astron Lett., 33, 771-779, 2007.

Wilson, I. R. G., Carter, B. D., and Waite, I. A.: Does a spin-orbit coupling between the sun and the jovian planets govern the solar cycle?, Publ. Astron. Soc. Aust., 25, 85-93, 2008.

Yang, D. H. and Yang, X. X.: Study and model on variation of Earth's Rotation speed, Prog Geophys., 28, 58-70, 2013.

Yang, R. W., Cao, J., Huang, W., and Nian, A. B.: Cross wavelet analysis of the relationship between total solar irradiance and sunspot number, Chin. Sci. Bull., 55, 2126-2130, 2010.

Yang, Z. G.: The possible influence of the orbital motion of the major planets on sunspot activities, Acta Astrophys. Sin., 11, 370377, 1991.

Yndestad, H. and Solheim, J.-E.: The influence of solar system oscillation on the variability of the total solar irradiance, New Astron., 51, 135-152, 2017.

Zhao, M. Y., Chen, J. Q., Liu, Y., Ibrahim, A., Yan, X. L., and Dun, J. P.: Statistical analysis of sunspot groups and flares for solar maximum and minimum, Sci. Sinica, 44, 109-120, 2014.

Zhao, X. H. and Feng, X. S.: Periodicities of solar activity and the surface temperature variation of the Earth and their correlations, Chin. Sci. Bull., 59, 1284-1292, 2014.

Zhao, P., Zhou, X. J., and Liu, G.: Decadal-centennial-scale change in Asian-Pacific summer thermal contrast and solar activity, Chin. Sci. Bull., 56, 3012-3018, 2011.

Zhou, Y. B.: The Course of Theoretical Mechanics (Second Edition), Beijing, Higher Education press, 2, 112-114, 1986. 